\title{
Why Does Every Hepatocellular Carcinoma Clinical Trial Using Molecular Targeted Agents Fail?
}

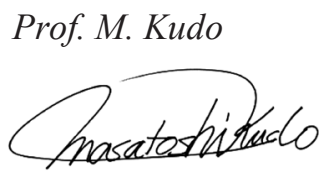

Editor Liver Cancer

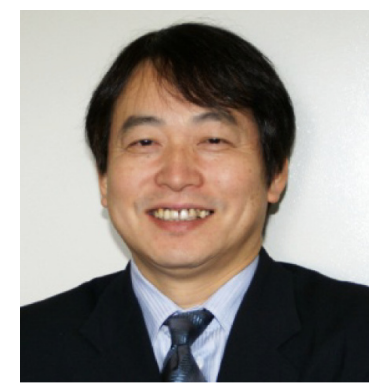

Sorafenib was approved for the treatment of unresectable hepatocellular carcinoma (HCC) in 2007 and is the first molecular targeted agent to be used for treating advanced HCC. It is also the only molecular targeted agent to have had a survival benefit demonstrated in two global phase III randomized controlled trials [1,2], and it has since been approved for use worldwide.

To date, more than 10 phase III global clinical trials of new molecular targeted agents have been undertaken or are currently ongoing. Of these, 8 have failed to meet their primary endpoint (as of August 2012).

The trials are the adjuvant Vitamin K2 study [3], the Japanese/Korean post-Transarterial Chemoembolization (TACE) study [4], the sunitinib first-line study [5], the adjuvant peretinoin (NIK333) study [6], the brivanib second-line study [7], the brivanib first-line study, the linifanib first-line study, and the SEARCH (Sorafenib and Erlotinib, a Randomized Trial Protocol for the Treatment of Patients with HCC) study, which is a phase III study of sorafenib plus erlotinib versus sorafenib plus placebo.

The problems experienced in the Japanese/Korean post-TACE study may be related to the study design (sequential design), to the treatment lag after TACE (9.3 weeks), or to the high early discontinuation rate (low drug exposure rate) [4]. Problems with the sunitinib first-line trial may be due to high toxicity, low tolerability, or to the high dose reduction and drug discontinuation rates (low drug exposure rate) [5]. In the adjuvant Peretinoin study, where Peretinoin was administered after curative treatment, problems may have arisen because the $300 \mathrm{mg}$ dose of Peretinoin failed to suppress HCC recurrence due to poor pharmacological activity. However, exploratory subset analysis revealed a positive signal for the $600 \mathrm{mg}$ dose of Peretinoin, showing a significant decrease in recurrence compared with the placebo [6]. Stage migration may be a problem with the brivanib second-line study because sorafenib treatment was evaluated in more advanced stage cancer in the two initial phase III studies [1, 2]. In other words, sorafenib was administered for intermediate-stage HCC in those trials, and post-trial therapies such as TACE or systemic/arterial infusion chemotherapy might have been administered to patients for whom second-line therapy had failed, thereby affecting the findings. However, the study clearly demonstrated improved antican- 
cer efficacy, as evaluated by tumor response rate and time to tumor progression. The problems with the linifanib first-line study, the brivanib first-line study, and the SEARCH study remain unknown. Details are expected to be presented at a forthcoming scientific meeting.

In conclusion, clinical trials of new molecular targeted agents for the treatment of HCC in an adjuvant setting, first-line setting, second-line setting, or in combination with TACE, seem to be experiencing difficulty and yielding poor results. These failures may be attributed to any of the following reasons. First, HCC is usually associated with impaired liver function or liver cirrhosis; therefore, toxicity or intolerability may be enhanced relative to patients with normal liver function, resulting in early discontinuation or dose reduction. Second, aside from resection, there are many effective treatment options for HCC including ablation, TACE [8], and hepatic arterial infusion chemotherapy [9]. Therefore, post-trial treatment might have affected the results of the primary endpoints such as overall survival.

There is a need for new molecular targeted agents for treating HCC that suppress recurrence after curative treatment or TACE as well as for agents for treating advanced HCC in patients with sorafenib resistance or intolerance. We look forward to positive findings from current or future trials of agents that can overcome the difficulties associated with HCC of the cirrhotic liver.

\section{References}

1 Llovet JM, Ricci S, Mazzaferro V, Hilgard P, Gane E, Blanc JF, et al: Sorafenib in advanced hepatocellular carcinoma. N Engl J Med 2008;359:378-390.

2 Cheng AL, Kang YK, Chen Z, Tsao CJ, Qin S, Kim JS, et al: Efficacy and safety of sorafenib in patients in the Asia-Pacific region with advanced hepatocellular carcinoma: a phase III randomised, double-blind, placebo-controlled trial. Lancet Oncol 2009;10:25-34.

3 Yoshida H, Shiratori Y, Kudo M, Shiina S, Mizuta T, Kojiro M, et al: Effect of vitamin K2 on the recurrence of hepatocellular carcinoma. Hepatology 2011;54:532-540.

4 Kudo M, Imanaka K, Chida N, Nakachi K, Tak WY, Takayama T, et al: Phase III study of sorafenib after transarterial chemoembolisation in Japanese and Korean patients with unresectable hepatocellular carcinoma. Eur J Cancer 2011;47:2117-2127.

5 Cheng AL, Kang YK, Lin DY, Park JW, Kudo M, Qin S, et al: Phase 3 trial of sunitinib versus sorafenib in advanced hepatocellular carcinoma. ASCO, 2011.

6 Okita K, Matsui O, Kumada H, Tanaka K, Kaneko S, Moriwaki H, et al: Peretinoin reduces recurrence of hepatocellular carcinoma: results of a phase II/III randomized placebo-controleed trial. ASCO, 2012.

7 Llovet JM, Decaens T, Raoul JL, Boucher E, Kudo M, Chang C, et al: Brivanib versus placebo in patients with advanced hepatocellular carcinoma (HCC) who failed or were intolerant to sorafenib: results from the phase III Brisk-Ps Study. EASL, 2012.

8 Lencioni R: Chemoembolization in patients with hepatocellular carcinoma. Liver Cancer 2012;1:41-50.

9 Kudo M: Treatment of advanced hepatocellular carcinoma with emphasis on hepatic arterial infusion chemotherapy and molecular targeted therapy. Liver Cancer 2012;1:62-70. 